\title{
Téoros
}

Revue de recherche en tourisme

\section{Le kayak en Wallonie}

À la croisée des enjeux du développement touristique et de la protection de l'environnement

\section{Bernard De Myttenaere et Emmanuel D’Ieteren}

Volume 28, numéro 2, 2009

Tourisme, sport et développement

URI : https://id.erudit.org/iderudit/1024802ar

DOI : https://doi.org/10.7202/1024802ar

Aller au sommaire du numéro

Éditeur(s)

Université du Québec à Montréal

ISSN

0712-8657 (imprimé)

1923-2705 (numérique)

Découvrir la revue

Citer cet article

De Myttenaere, B. \& D’Ieteren, E. (2009). Le kayak en Wallonie : à la croisée des enjeux du développement touristique et de la protection de l'environnement. Téoros, 28(2), 9-20. https://doi.org/10.7202/1024802ar
Résumé de l'article

Depuis une trentaine d'années, les activités ludosportives en milieu rural occupent une place non négligeable au sein de nombreux territoires touristiques, témoignant ainsi d'un nouveau rapport au temps, au corps et à la nature au sein de la société occidentale. Parmi ces activités, le kayak interpelle nombre d'acteurs publics et privés liés à la gestion du milieu aquatique, de par les enjeux socio-économiques, culturels et environnementaux, et les conflits que cette activité suscite. En Wallonie, comme dans de nombreux territoires ruraux, la rivière est ainsi le théâtre de conflits d'usage qui opposent des groupes d'acteurs aux valeurs et intérêts souvent divergents. Dans un contexte de montée en puissance des préoccupations environnementales, force est de constater que la pratique du kayak a été touchée en Wallonie par de nombreuses réglementations et soumise à toujours davantage de contraintes visant à minimiser les supposées incidences de cette activité. Cependant, l'adoption de ces mesures, considérées comme illégitimes par les loueurs de kayaks, conjuguée à l'absence d'étude objectivant les impacts environnementaux de la pratique du kayak ont participé à l'éclosion d'une importante controverse environnementale.

Partant de ce constat, cette contribution présente, d'une part, les résultats d'une étude visant à mieux comprendre les spécificités socio-économiques de la pratique du kayak en Wallonie et, d'autre part, montre les enjeux auxquels sont confrontées les activités sportives de loisir et de tourisme de nature en Wallonie. Dans notre analyse, nous exposons une série de facteurs qui, à nos yeux, expliquent l'existence d'une controverse environnementale et notamment le jeu de rapports de force inégaux entre les différents acteurs de la rivière. L'étude montre les difficultés que rencontre la filière des sports de nature en Wallonie dans un contexte où les modes de régulation de l'activité du kayak concilient difficilement les enjeux du développement du tourisme sportif de nature et la protection de l'environnement.
Ce document est protégé par la loi sur le droit d'auteur. L’utilisation des services d’Érudit (y compris la reproduction) est assujettie à sa politique d'utilisation que vous pouvez consulter en ligne.

https://apropos.erudit.org/fr/usagers/politique-dutilisation/ 


\section{Le kayak en Wallonie À la croisée des enjeux du développement touristique et de la protection de l'environnement}

\author{
Bernard DE MYTTENAERE ${ }^{1}$ \\ Assistant au Laboratoire \\ Interdisciplinaire Tourisme, Territoire, Sociétés \\ Institut de Gestion de I'Environnement \\ et d'Aménagement du Territoire \\ Université Libre de Bruxelles \\ bdemytte@ulb.ac.be
}

\author{
Emmanuel D'IETEREN \\ Collaborateur scientifique au Laboratoire \\ Interdisciplinaire Tourisme, Territoire, Sociétés \\ Institut de Gestion de I'Environnement \\ et d'Aménagement du Territoire \\ Université Libre de Bruxelles \\ edieter@ulb.ac.be
}

RÉSUMÉ: Depuis une trentaine d'années, les activités ludosportives en milieu rural occupent une place non négligeable au sein de nombreux territoires touristiques, témoignant ainsi d'un nouveau rapport au temps, au corps et à la nature au sein de la société occidentale. Parmi ces activités, le kayak interpelle nombre d'acteurs publics et privés liés à la gestion du milieu aquatique, de par les enjeux socio-économiques, culturels et environnementaux, et les conflits que cette activité suscite. En Wallonie, comme dans de nombreux territoires ruraux, la rivière est ainsi le théâtre de conflits d'usage qui opposent des groupes d'acteurs aux valeurs et intérêts souvent divergents. Dans un contexte de montée en puissance des préoccupations environnementales, force est de constater que la pratique du kayak a été touchée en Wallonie par de nombreuses réglementations et soumise à toujours davantage de contraintes visant à minimiser les supposées incidences de cette activité. Cependant, l'adoption de ces mesures, considérées comme illégitimes par les loueurs de kayaks, conjuguée à l'absence d'étude objectivant les impacts environnementaux de la pratique du kayak ont participé à l'éclosion d'une importante controverse environnementale.

Partant de ce constat, cette contribution présente, d'une part, les résultats d'une étude visant à mieux comprendre les spécificités socio-économiques de la pratique du kayak en Wallonie et, d'autre part, montre les enjeux auxquels sont confrontées les activités sportives de loisir et de tourisme de nature en Wallonie. Dans notre analyse, nous exposons une série de facteurs qui, à nos yeux, expliquent l'existence d'une controverse environnementale et notamment le jeu de rapports de force inégaux entre les différents acteurs de la rivière. L'étude montre les difficultés que rencontre la filière des sports de nature en Wallonie dans un contexte où les modes de régulation de l'activité du kayak concilient difficilement les enjeux du développement du tourisme sportif de nature et la protection de l'environnement.

Mots-clés: Tourisme et sports de nature, kayak, Wallonie, conflits d'usage, régulation, controverse environnementale.

Depuis une trentaine d'années, les activités ludosportives en milieu rural occupent une place de plus en plus importante au sein de nombreux territoires touristiques. Situées à la croisée d'enjeux socio-économiques et environnementaux, ces activités interpellent toujours davantage les acteurs publics et privés liés à la protection et à la valorisation de l'espace rural. En effet, peu de territoires ruraux voulant s'inscrire dans des politiques de développement durable échappent aux problématiques suscitées par le difficile équilibre entre le développement économique local et la protection de l'environnement. Une illustration symptomatique de ces enjeux territoriaux nous est donnée par la pratique touristique du kayak en Wallonie. En effet, les années 1990 et 2000 ont été caractérisées par la prise de diverses mesures réglementaires en vue de restreindre les espaces et les périodes de la pratique touristique du kayak, accusée de plusieurs maux environnementaux (destruction de la faune benthique, destruction des berges, production de déchets, nuisance sonore, etc.) et, dès lors, considérée comme étant peu compatible avec les politiques de protection de l'environnement. Ces mesures, nées de tensions entre des pêcheurs et des kayakistes qui ont une représentation divergente et des usages différents des cours d'eau, n'ont pas été sans conséquences pour cette filière qui peine à se structurer et à s'organiser.

Centrée sur la Wallonie, la présente contribution propose, d'une part, de présenter les résultats d'une étude visant à 
mieux comprendre les outils de régulation et les spécificités socio-économiques de la pratique du kayak et, d'autre part, de montrer les enjeux auxquels sont confrontées les activités sportives de loisir et de tourisme dans ce territoire. Prenant comme point de départ une législation adoptée en 2000 relative à la pratique du kayak, nous identifierons et analyserons certains facteurs qui, selon nous, expliquent l'existence d'une controverse environnementale autour de la pratique du kayak en Wallonie, ainsi que le jeu de rapports de force inégaux entre les différents acteurs de la rivière. Cette étude de cas nous permettra de montrer les difficultés que rencontre le développement des sports de nature en Wallonie dans un contexte où les modes de régulation de l'activité du kayak concilient difficilement les enjeux du développement du tourisme sportif de nature et la protection de l'environnement.

\section{Les activités sportives de loisir et de tourisme en Wallonie}

L'émergence des activités sportives et touristiques en milieu naturel constitue une étape importante de l'évolution de la société de loisirs. Plusieurs facteurs vont contribuer à l'essor de ces activités, tels que la réduction progressive du temps de travail, l'avènement de la société de consommation ainsi que les nouveaux rapports à la nature entretenus par les sociétés urbaines. Les activités physiques de pleine nature se développent particulièrement durant les années 1980-90 «à la faveur d'une évolution des valeurs qui privilégient un nouveau rapport à soi, aux autres et à la nature» (Vigarello, 1982 dans Bessy et Mouton, $2004: 15)$.

La massification de nouvelles pratiques sportives de pleine nature, telles que le kayak, le canoë ou le VTT, et la place de plus en plus importante que ces pratiques ont pris au sein des activités de loisirs, ont entraîné dans leur sillage le développement d'un véritable marché. Issus notamment des associations sportives, des acteurs se sont ainsi mobilisés pour inscrire des activités relevant à l'origine du passe-temps dans une démarche de commercialisation (Fontugne, $2004: 16$ ). Ainsi que Corneloup et al. (2001:21) l'observent, la multiplication des prestataires, des produits sportifs et des aménagements d'espaces de nature démontre que le tourisme sportif de nature est un secteur en pleine croissance. Les sports de nature sont ainsi devenus une composante importante de l'offre touristique de nombreux territoires et peuvent, à ce titre, être considérés comme de réels vecteurs de structuration des territoires (Bessy et Mouton, $2004: 15$ ).

Cette observation est également valable en ce qui concerne le cas de la Wallonie, qui a connu ces trente dernières années un engouement pour les activités sportives et touristiques en milieu rural. Ce territoire de près de $17000 \mathrm{~km}^{2}$ situé dans le sud de la Belgique et comptant près de 3500000 habitants occupe, de par ses caractéristiques géographiques, historiques et socioculturelles, une place très modeste mais stable au sein des destinations touristiques mondiales. De fait, disposant d'environ 70000 lits dans plus de 4000 hébergements de tous types (campings, hôtels, gîtes ruraux), la Wallonie a accueilli en 2006 environ 2,6 millions de touristes, totalisant plus de 6,8 millions de nuitées (Cellule état de l'environnement wallon, 2008).

À l'instar d'autres destinations touristiques européennes, on observe en Wallonie, ces dernières années, une diminution du nombre de nuitées parallèlement à une augmentation du nombre d'arrivées, entraînant ainsi une chute de la durée moyenne de séjour. En 2006, les attractions touristiques wallonnes ont attiré, quant à elles, environ 8,25 millions de touristes

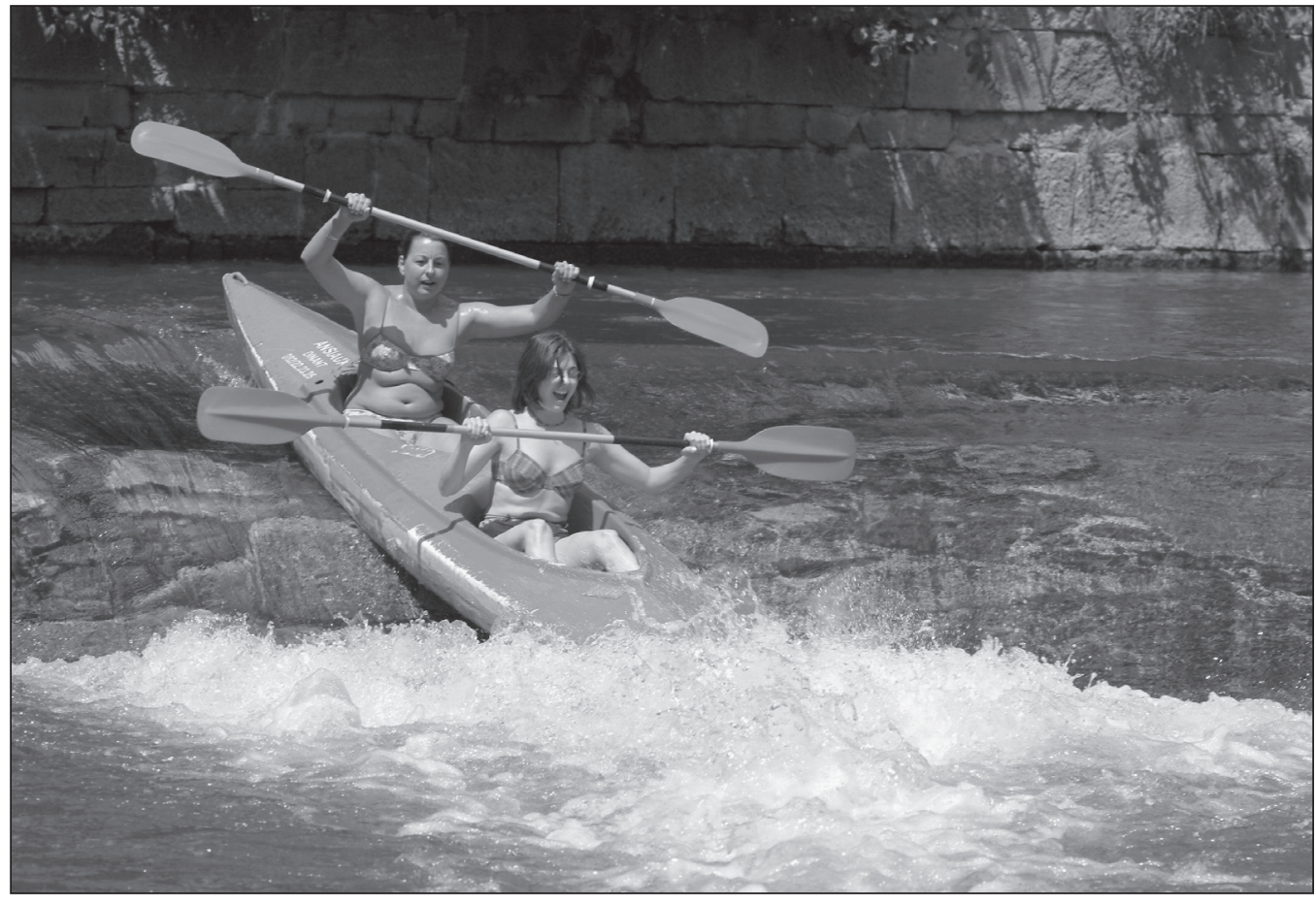

ILLUSTRATION 1 : La descente de la Lesse en kayak est une activité récréative et peu technique (photo : OPT Wallonie-Bruxelles/J.-L. Flémal). 
et excursionnistes, dont $30 \%$ dans les centres récréatifs et les parcs d'attractions, $22 \%$ dans les musées, $20 \%$ dans les attractions naturelles et $11 \%$ dans les attractions nautiques (Cellule état de l'environnement wallon, 2008).

Alors que dans les deux autres régions belges (la Région flamande et la Région bruxelloise), le tourisme est davantage urbain, la Wallonie, occupée à $80 \%$ par des terres agricoles et boisées, est un territoire où le tourisme rural et de nature est une caractéristique forte de l'offre et de la demande. Parallèlement à la structuration des associations et fédérations sportives locales et régionales, les sports de nature y ont progressivement fait l'objet de processus de valorisation commerciale et touristique venant ainsi compléter les structures associatives de sports de nature (IEW, 2000a). Si le territoire wallon n'a pas la superficie ni la diversité topographique et paysagère de son voisin français, plusieurs sports de nature s'y sont toutefois bien développés, comme le VTT, la spéléologie, le parapente et le kayak (Jouret, 2000 : 29).

\section{Le kayak en Wallonie : une activité de loisir et une composante de l'offre touristique}

De par sa faible technicité par rapport à d'autres sports et son ancienneté dans l'émergence des sports de nature, la pratique du kayak est une des activités emblématiques ayant participé au développement des activités sportives et touristiques en milieu naturel de différentes destinations wallonnes (illustration 1).

La première société de location de kayaks a été créée sur la Lesse en 1921 et ce développement est resté essentiellement localisé sur ce cours d'eau jusqu'au milieu des années 80 . De nombreuses sociétés de location se sont ensuite établies dans d'autres espaces ruraux de la Wallonie, exploitant de nouveaux tronçons et mettant en circulation un nombre croissant d'embarcations. C'est dans les années 1985 à 1990 que le kayak va connaître un engouement important suite, notamment, à une politique de promotion très intensive de l'activité menée par les principales sociétés de location et à l'essor du tourisme d'un jour et des activités de nature.

Les cours d'eau accessibles à la pratique du kayak La Wallonie compte plus de 12000 cours d'eau totalisant environ $24000 \mathrm{~km}$ et répartis selon plusieurs catégories et niveaux de compétences (tableau 1).

À l'heure actuelle, la pratique du kayak n'est autorisée que sur les voies navigables et sur une partie des cours d'eau non navigables de première catégorie. Plus précisément, sur les cours d'eau non navigables, l'ensemble des embarcations à moteur est interdit et, parmi les embarcations dépourvues de moteur, seuls les barques de pêche, les kayaks, canoës et embarcations gonflables, ainsi que les embarcations utilisées à des fins récréatives sont acceptés.

\section{Les pratiquants du kayak}

Traditionnellement, on distingue quatre catégories d'acteurs pour les sports de nature en Wallonie : les fédérations sportives, les particuliers, les entreprises de location et les associations. Concernant le kayak, trois de ces quatre catégories d'acteurs apparaissent avec des profils et des modalités de pratique différents : les kayakistes sportifs, regroupés au sein de la Fédération francophone de canoë (FFC), les kayakistes privés et les entreprises commerciales de location de kayaks. Ces dernières peuvent être scindées en deux sous-groupes : les kayakistes "enregistrés », dont une part non négligeable est membre de la Fédération des loueurs de kayaks en Wallonie (FLKW), et les «entreprises pirates».

La FFC, la branche francophone de la Fédération royale belge de canoë (FRBC), regroupe près de 500 membres répartis dans une vingtaine de clubs sur l'ensemble du territoire wallon. Ces membres, qui possèdent tous leur propre kayak ainsi qu'une licence, effectuent en moyenne une sortie tous les

\begin{tabular}{|c|c|c|c|}
\hline \multicolumn{4}{|c|}{ Tableau 1 : Catégories de cours d'eau en Wallonie } \\
\hline Catégorie de cours d'eau & Réseau & Administration gestionnaire & Pratique du kayak \\
\hline Voies navigables & $740 \mathrm{~km}$ & $\begin{array}{l}\text { Administration de la Mobilité } \\
\text { et des Voies Hydrauliques }\end{array}$ & Autorisée \\
\hline $\begin{array}{l}\text { Cours d'eau non navigables } \\
\text { de } 1^{\text {re }} \text { catégorie } \\
\text { (bassin hydrographique } \\
\text { supérieur à } 5000 \text { ha) }\end{array}$ & 1651 km & $\begin{array}{l}\text { Administration de l'Agriculture, des Ressources } \\
\text { Naturelles et de l'Environnement, Direction } \\
\text { des Cours d'Eau Non Navigables }\end{array}$ & Autorisée partiellement \\
\hline $\begin{array}{l}\text { Cours d'eau non navigables } \\
\text { de } 2^{e} \text { catégorie } \\
\text { (bassin hydrographique } \\
\text { inférieur à } 5000 \text { ha) }\end{array}$ & \multirow{2}{*}{$15749 \mathrm{~km}$} & Provinces & Non autorisée \\
\hline $\begin{array}{l}\text { Cours d'eau non navigables } \\
\text { de } 3^{\mathrm{e}} \text { catégorie } \\
\text { (bassin hydrographique } \\
\text { inférieur à } 5000 \text { ha) }\end{array}$ & & Communes & Non autorisée \\
\hline $\begin{array}{l}\text { Cours d'eau non classés } \\
\text { (bassin hydrographique } \\
\text { inférieur à } 100 \text { ha) }\end{array}$ & 4000 km & Propriétaires riverains & Non autorisée \\
\hline
\end{tabular}


15 jours. Se portant garante du bon comportement de ses pratiquants, la Fédération francophone de canoë obtient souvent des dérogations auprès de l'administration en charge de la gestion écologique du milieu naturel (la Division de la Nature et des Forêts) pour permettre à ses membres de naviguer sur une série de petites rivières qui sont habituellement interdites au grand public. Outre cette dérogation spéciale, ses membres peuvent également profiter toute l'année de quatre rivières normalement interdites à la navigation.

Plus numériquement marginaux, les kayakistes privés sont des personnes qui pratiquent le kayak à titre individuel sans passer par l'intermédiaire d'entreprises commerciales. Répertorier l'ensemble de ces personnes pose problème, car elles ne s'inscrivent dans aucune structure officielle et ne payent pas de taxe communale, qui ne concerne que les embarcations de descente de cours d'eau utilisées dans un but lucratif.

Ce sont les clients des sociétés commerciales de location de kayaks qui constituent la grande majorité des pratiquants. Deux types d'entreprises se partagent le marché : d'une part, les sociétés commerciales reconnues, dont une majeure partie est membre de la Fédération des loueurs de kayaks en Wallonie (FLKW) et, d'autre part, les «entreprises pirates».

Créée en 1993, la FLKW regroupe tous les loueurs qui payent leurs cotisations annuelles. En 2003, ils étaient 24 membres sur environ 30 entreprises qui travaillent sur l'Amblève, l'Our, l'Ourthe, la Semois, la Lesse et le Viroin. Cette fédération a pour but de rassembler les différents loueurs de kayaks afin, notamment, de leur permettre d'avoir un certain poids dans les négociations avec les instances en charge des différentes réglementations liées à la pratique de cette activité (circulation sur les cours d'eau, aires d'embarquement et de débarquement, taxes, etc.).

Quant aux «entreprises pirates», il s'agit de sociétés de location de kayaks qui fréquentent les cours d'eau de Wallonie en venant avec leurs propres embarcations et clients, mais qui ne payent ni taxe communale ni redevance pour l'activité organisée sur les cours d'eau. Cette situation non réglementaire leur permet de proposer des prix plus attractifs que ceux appliqués par les loueurs officiels. Cette concurrence déloyale génère un manque à gagner non seulement pour les entreprises de locations enregistrées, mais également pour les communes concernées par ces activités. Notons, à cet égard, qu'en 2003, plus de $450000 €$ de taxes ont été rétribués aux pouvoirs publics. L'administration a conscience de ce problème et parle d'un manque de moyens législatifs pour lutter contre cette situation. En effet, si ces «entreprises pirates» embarquent dans les aires d'embarquement officielles aux horaires adéquats, l'administration n'est pas en mesure de les sanctionner au regard de la législation en vigueur.

\section{L'offre commerciale}

À l'instar d'autres activités récréatives et touristiques, les sources de données socio-économiques concernant le secteur du kayak sont peu nombreuses et émanent bien souvent directement des entreprises elles-mêmes, ce qui implique dès lors une exploitation et une interprétation prudentes de ces données. Il n'existe à ce jour aucune statistique officielle reprenant des données sur les entreprises de location de kayaks, ce qui reflète le peu d'intérêt porté à cette problématique en Wallonie ainsi que les difficultés d'organisation de ce secteur.

Néanmoins, quelques données fragmentaires ne concernant que les sociétés affiliées et domiciliées le long des tronçons exploités ont pu être recueillies et corroborées, entre autres, auprès de la Fédération des loueurs de kayaks en Wallonie (FLKW) et de l'administration régionale. Selon De Myttenaere et al. (2007 : 117), environ 7000 embarcations ont été déclarées en 2003 par l'ensemble des sociétés de location.

Par ailleurs, selon la FLKW (Wilputte, 2003 : 21), la fréquentation annuelle du kayak en Wallonie est estimée, pour 2003, à environ 700000 descentes par an avec un chiffre d'affaires annuel de 12,5 millions d'euros. Les entreprises de location reconnues emploieraient de 200 à 300 personnes à temps plein et de 1400 à 1800 saisonniers. En dehors du coût de la location du kayak, les touristes d'un jour dépenseraient en moyenne 25 euros par jour alors que les touristes de séjour dépenseraient de 40 à 45 euros par jour. L'impact total global sur les régions fréquentées par les kayakistes serait de plus de 20 millions d'euros. Ce montant comprend les prestations liées à la restauration, à l'hébergement et autres activités complémentaires pratiquées durant le séjour des kayakistes (Wilputte, $2003: 21$ ).

\section{Caractéristiques socio-économiques des loueurs de kayaks}

En vue de mieux connaître le profil des entreprises de location, une enquête téléphonique a été menée auprès de loueurs de kayaks en Wallonie entre novembre et décembre 2006 sur la base d'entretiens semi-directifs. Les objectifs de cette enquête étaient principalement de mieux comprendre le fonctionnement de l'activité de location de kayaks et de mieux saisir les perceptions que les loueurs se font de cette activité et de son devenir. L'enquête portait sur les caractéristiques socioéconomiques des propriétaires des sociétés de location ainsi que sur les sociétés de location proprement dites. Sur un total de 58 sociétés de location de kayaks répertoriées, 30 sociétés ont répondu à notre enquête. Les informations récoltées lors de ces interviews ont été exploitées avec l'aide du logiciel de traitement d'enquête Sphinx Lexica.

L'âge des gestionnaires est en moyenne de 44 ans. Leur niveau d'étude équivaut pour $37 \%$ d'entre eux au cycle secondaire et, pour $53 \%$ des loueurs, il correspond à des études supérieures (dont $20 \%$ en supérieur universitaire). $46 \%$ des personnes interrogées sont issues du secteur du tourisme (secteur du kayak compris) et $33 \%$ des personnes interrogées sont originaires du secteur du kayak uniquement.

L'ancienneté des sociétés de location de kayaks est, en moyenne, de 20 ans. La plus ancienne date de 1949 et la dernière a été créée en 2003. L'enquête nous apprend que $43 \%$ de ces sociétés ont été créées après 1990.

Le nombre moyen de kayaks par exploitation est d'environ 150 unités avec un minimum de 15 et un maximum de 900 kayaks par exploitation. Près de $90 \%$ des exploitations comptent moins de 200 kayaks. Seuls quelques loueurs dépassent les 500 embarcations, ce qui est par exemple le 
cas des trois loueurs actifs sur la basse Lesse qui totalisent déjà plus de 2000 kayaks à eux seuls. Même si le nombre de kayaks ne définit pas la capacité maximale d'accueil par jour, puisqu'un certain nombre de ces embarcations sont biplaces et qu'un kayak peut être reloué jusqu'à trois fois sur une même journée, ces données mettent néanmoins en évidence l'existence de grands "loueurs» (plus de 500 kayaks) et de "petits» loueurs. Cette polarisation n'est pas sans conséquences sur l'organisation de l'activité comme nous le verrons dans l'analyse des conflits d'usage.

De par le climat de la Belgique, la location de kayak à des fins de loisir y est une activité saisonnière. De plus, le kayak est un produit assez instable en raison d'une météo variable, ce qui explique que $80 \%$ des sociétés proposent également plusieurs activités et services autres que le kayak (sports d'aventure, restauration, hébergement, autres sports, activités non touristiques). Plusieurs petits loueurs éprouvent des difficultés pour se diversifier, notamment par la location de vélos, car cette activité requiert des compétences techniques qu'ils ne considèrent pas posséder. Les loueurs ne diversifiant pas leur activité ont souvent pour projet de terminer leur carrière, car ils ne sont pas prêts à investir lourdement dans cette activité et aucune reprise de cette dernière n'est envisagée.

\section{La réglementation de la pratique du kayak en Wallonie : des mesures contraignantes et sujettes à polémique}

C'est au milieu des années 1970 que le monde de la pêche va entreprendre de multiples démarches pour obtenir une réglementation de la pratique du kayak. Durant cette période, qui correspond aux prémices du développement de la pratique touristique du kayak, cette activité entre déjà en conflit avec les usages plus traditionnels de la rivière. Ce n'est qu'en 1980 qu'une première réglementation portant sur les cours d'eau non navigables de première catégorie interdit la circulation des kayaks, entre $17 \mathrm{~h}$ et $10 \mathrm{~h}$, durant le printemps et l'été. En 1985, une nouvelle réglementation confirmera ces dispositions et les complètera par l'interdiction de la circulation des kayaks les jours d'ouverture de la pêche. Historiquement, la réglementation de l'activité du kayak a ainsi été initiée en Wallonie en vue de tenir compte prioritairement des intérêts des pêcheurs (encadré 1 ).

En 1994, la Région wallonne adopte une nouvelle réglementation portant sur la circulation des bateaux de plaisance, et des kayaks en particulier. D'emblée, la protection de l'environnement en constitue le leitmotiv avancé puisque le cadre réglementaire repose sur la loi belge sur la conservation de la nature de 1973. Plus précisément, comme Gérard et De Bast (2000 : 139) le développent, la nouvelle législation répond à la volonté régionale de limiter les conflits entre les différents usages des rivières, l'exemple le plus emblématique de la tension entre pêcheurs, praticiens du kayak et conservation de la nature étant celui de la rivière Lesse.

En vue de renforcer les mesures de restriction de la circulation des kayaks, la réglementation de 1994 a été amendée à plusieurs reprises depuis son adoption (en 1996, en 2000 et en 2001), donnant lieu notamment à la limitation des tronçons de cours d'eau où l'activité est autorisée. Les cinq cours

\section{ENCADRÉ 1 : Compétences partagées en Belgique}

La Belgique est un état fédéral composé de trois régions et trois communautés linguistiques. Dans ce cadre institutionnel, les compétences du tourisme, de l'environnement, de la conservation de la nature et de l'aménagement du territoire, essentielles à la bonne gestion des activités nautiques en milieu naturel, relèvent des entités fédérées que sont la Région wallonne, la Région de Bruxelles-Capitale et la Région flamande. À ce titre, les différentes directives européennes relatives à l'environnement, la gestion de la nature et la gestion des cours d'eau doivent être transposées et appliquées par les différentes régions. Dès lors, sur le territoire wallon, la gestion de la circulation des kayaks est une matière qui repose sur les mesures élaborées ou transposées par l'institution régionale.

d'eau concernés - le Viroin, l'Ourthe, l'Amblève, la Lesse et la Semois - et les tronçons de rivière accessibles aux kayaks sont mis en évidence sur l'illustration 2.

Reposant sur des listes d'exclusion de cours d'eau et des débits de référence, les restrictions adoptées pour équilibrer les intérêts des différents usages de la rivière traduisent le modèle de "gestion environnementale autoritaire» (versus gestion concertée) tel que décrit par Mounet (2000a : 3).

Tout d'abord, la réglementation distingue trois catégories de cours d'eau en rapport avec la circulation des embarcations de loisirs : la première catégorie rassemble les cours d'eau sur lesquels la circulation est autorisée toute l'année selon des horaires précis; la deuxième comprend les cours d'eau sur lesquels la circulation des embarcations n'est autorisée que du $1^{\text {er }}$ octobre au 15 mars inclus; enfin, la troisième catégorie correspond aux cours d'eau où la circulation est totalement interdite. La circulation des kayaks est donc réglementée par des restrictions saisonnières, affectant la haute saison touristique, et par des plages horaires en vue de dégager des périodes d'usage exclusif de la rivière pour les amateurs de la pêche.

Complémentairement à cette classification, la Région wallonne s'est également réservé, depuis 1996, la possibilité d'interdire temporairement la circulation des kayaks sur les cours d'eau où cela est normalement autorisé, au titre de la conservation de la nature. Ces interdictions sont appliquées dès que des seuils de débits d'eau minimaux et maximaux sont atteints, dans le but d'éviter que les kayaks ou autres embarcations de loisirs ne circulent lorsque le niveau d'eau est trop faible ou trop important, réduisant ainsi les risques de dommages environnementaux dans les milieux aquatiques (au niveau de la végétation, des sédiments, de la faune benthique...). Par ailleurs, ces seuils ont été établis pour chaque cours d'eau et différenciés par tronçon (Gérard et De Bast, 2000 : 140).

De nombreuses entreprises de location de kayaks seront directement touchées par ces nouvelles mesures d'interdictions temporaires, mais il faut cependant attendre l'adoption en 2000 de nouvelles mesures de restriction pour observer de très vives réactions du secteur touristique et l'expression d'une forte polémique. En effet, suite aux décisions prises par le Gouvernement wallon, plusieurs tronçons de différentes 


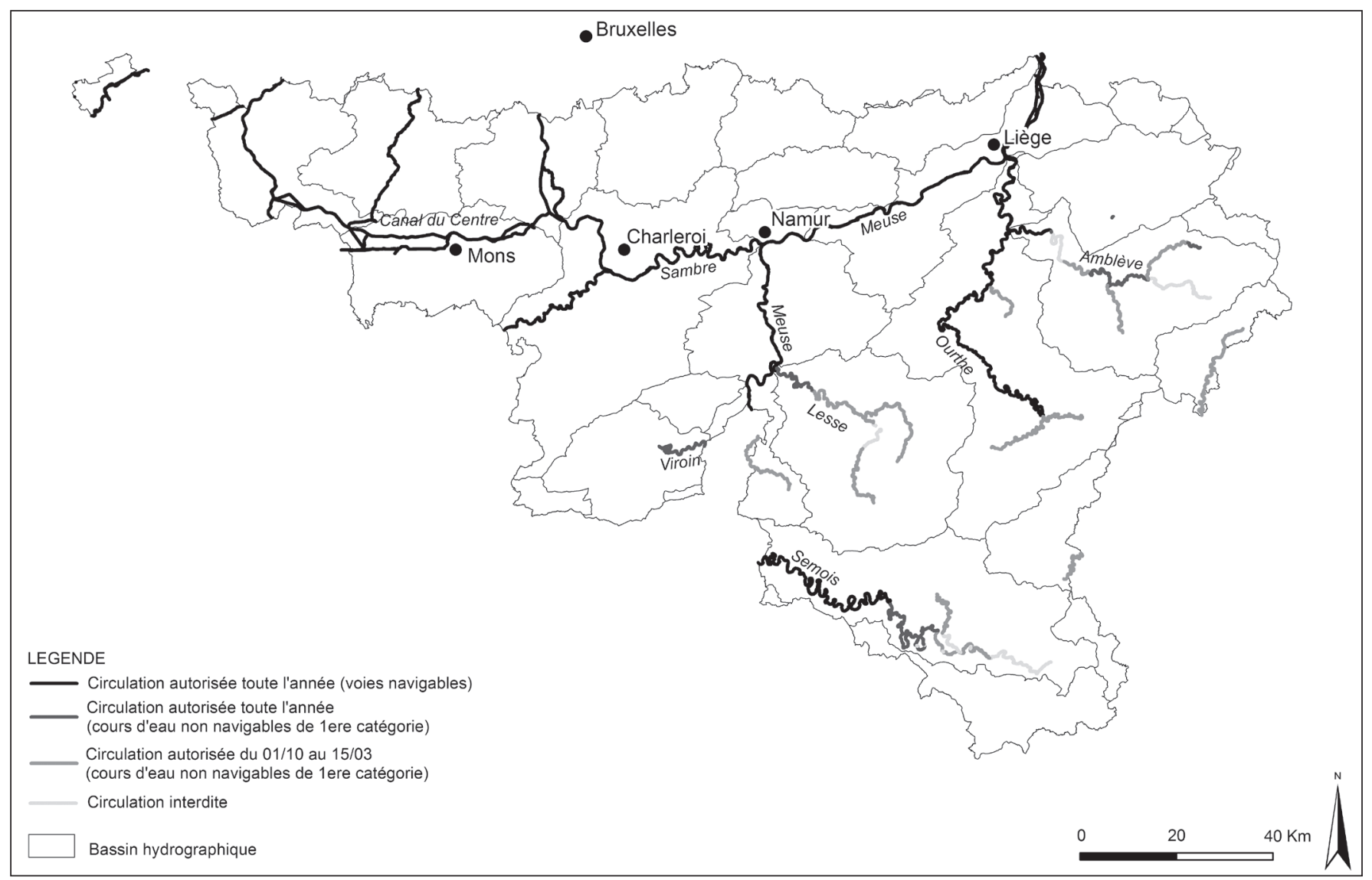

ILLUSTRATION 2 : Tronçons de cours d'eau accessibles au kayak en Wallonie (source : RW, non daté, Internet et DGARNE, 2009, communications personnelles).

rivières (Lesse, Amblève, Our, Ourthe, Warche) vont rejoindre la liste des cours d'eau interdits à la circulation des kayaks durant la période de mars à octobre. Ensuite, certains débits seuils ont été revus à la baisse. Comme nous le verrons plus loin, ces nouvelles dispositions seront très mal acceptées par les loueurs de kayaks voyant en celles-ci une menace particulièrement forte pour leur activité commerciale. De par les mesures de régulation adoptées, la pratique du kayak en Wallonie est ainsi située au carrefour des enjeux du développement du tourisme de nature et de la protection de l'environnement (illustration 3).

\section{Une forte controverse}

\section{autour de la réglementation de 2000}

Partant du postulat que les conflits s'expriment bien souvent en dehors du premier cercle des acteurs sociaux directement concernés par un litige et s'extériorisent notamment par la presse (Torre et Lefranc, 2006 : 95), nous avons privilégié comme méthode de travail l'analyse de la presse écrite pour rendre compte et comprendre les tensions existant entre le monde du kayak et celui des pêcheurs en Wallonie. En effet, les acteurs d'un conflit utilisent régulièrement la presse afin de sensibiliser l'opinion publique et d'exercer par ce biais des pressions pour tenter d'obtenir, par exemple, une modification des règles d'arbitrage en vigueur (Paoli, 2009).
Reposant sur l'analyse d'une cinquantaine d'articles de la presse locale, régionale et nationale relatant les conflits spécifiquement liés à la pratique du kayak, cette partie a donc pour objectif d'illustrer la polémique suscitée par l'adoption de la réglementation de 2000 relative à la circulation des kayaks sur les cours d'eau wallons. Cette analyse vise plus spécifiquement à mieux définir la position exprimée par les différents acteurs concernés, à savoir : le ministre à l'origine de la réglementation, les loueurs de kayak s'exprimant par la voix de leur fédération, les pêcheurs et, enfin, les associations environnementales.

Le ministre à l'origine de la réglementation est le ministre Happart, en charge de l'environnement entre 1994 et 1999, et de la ruralité et de la conservation de la nature de 1999 à 2004. Son point de vue à l'égard des rapports entre l'environnement et le tourisme de plein air est empreint de présupposés. Selon lui, le tourisme de plein air est source de perturbations importantes «sur le patrimoine naturel, et la modification envisagée [réglementation de 2001] vise à préserver les biotopes les plus fragiles contre le passage incessant d'embarcations et les accostages répétés, incités par les échouages trop fréquents » (Haquin, $2000: 21$ ). Il postule que l'activité du kayak est automatiquement néfaste pour les biotopes et envisage dès lors un lien de causalité directe entre la pratique du kayak, les cours d'eau en tant que milieux récepteurs et une pression négative qui résulterait immanquablement de cette relation. 


ILLUSTRATION 3 : Le kayak
en Wallonie, une pratique
au carrefour des enjeux
de développement du tourisme
de nature et de la protection
de l'environnement
(photo : OPT Wallonie-Bruxelles/
J.-L. Flémal).

ILLUSTRATION 3 : Le kayak en Wallonie, une pratique au carrefour des enjeux de développement du tourisme de nature et de la protection (photo : OPT Wallonie-Bruxelles/ J.-L. Flemal).

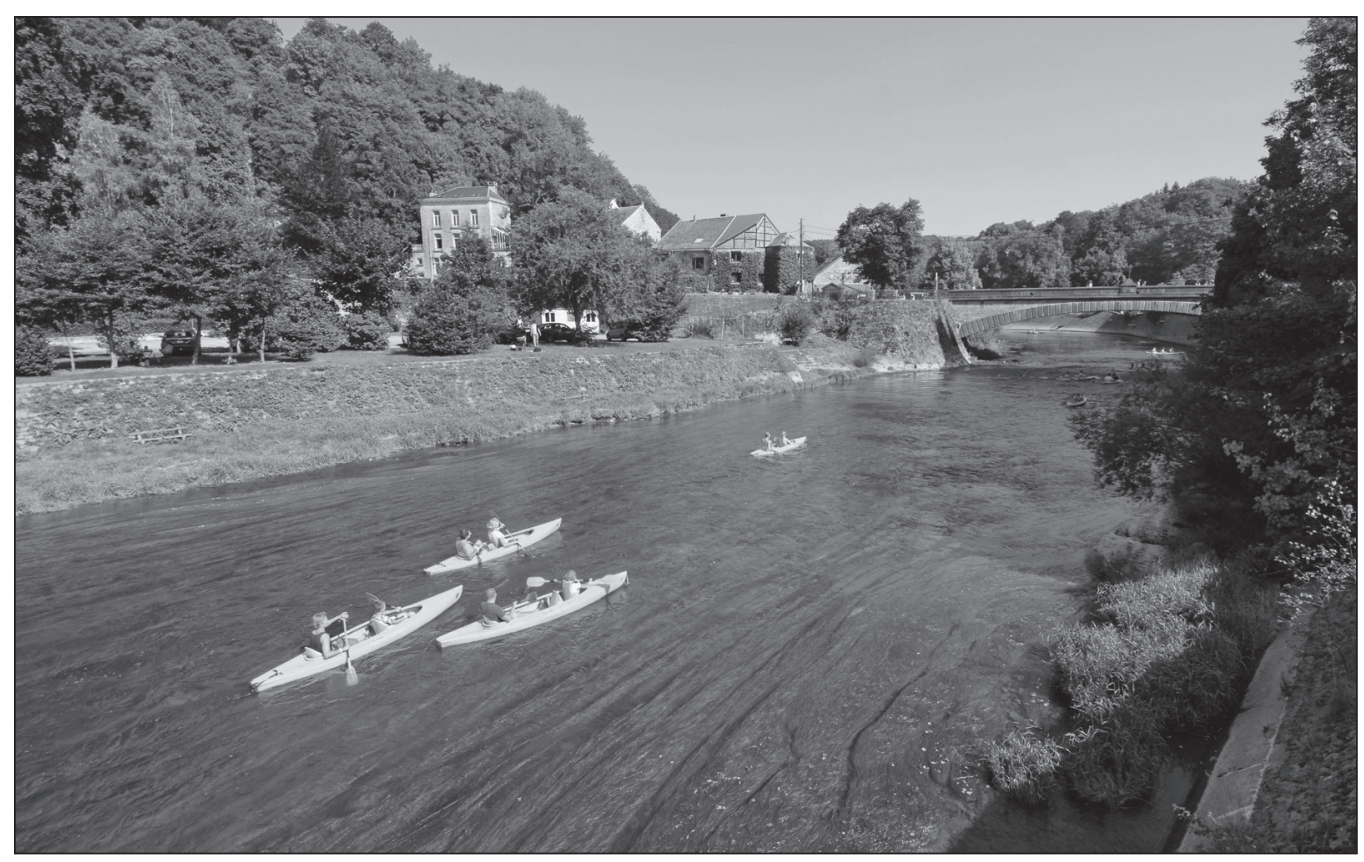

«Lorsqu'elles sont confinées et pratiquées par un nombre élevé de personnes, [elles] entraînent immanquablement une dégradation des biotopes aquatiques, de la flore, tant aquatique que rivulaire, et un dérangement des espèces animales, lequel peut notamment compromettre leur reproduction" (Note du ministre Happart au gouvernement wallon, 26 mai 2000). Il ressort clairement de ces quelques lignes une stigmatisation des effets potentiels (toujours perçus comme négatifs) de l'activité touristique sur l'environnement.

En tant qu'acteurs faisant un usage commercial de la rivière, les loueurs de kayaks, par la voix de leur fédération, ont fortement contesté les nouvelles mesures de restriction des tronçons ouverts à la circulation des kayaks. Selon eux, ces mesures vont «tuer le secteur» (Dorzee, 2000a : 19), provoquer «la mort économique du secteur» (Dorzee, 2000b : 17), car «la moitié des membres de notre fédération est menacée» (De Gendt, 2000 : 4). Ils craignent en effet une diminution forte du chiffre d'affaires et du nombre d'emplois. Ils évaluent la perte d'activité à $50 \%$ si le tronçon navigable est réduit de $100 \mathrm{~km}$ compte tenu des mesures prises. Par ailleurs, ils démontrent l'importance des enjeux économiques de cette nouvelle réglementation en invoquant un chiffre d'affaires global de 15 millions d'euros, plusieurs centaines d'emplois directs (environ 350) ainsi que des retombées indirectes non négligeables pour les acteurs locaux (taxes communales, commerces, restaurants, etc.). Les loueurs de kayaks contestent également «l'absence de dialogue», le «manque de concertation» avec le cabinet du Ministre qui affirme, de son côté, les «avoir rencontrés» et qui souligne le problème que les loueurs «ne parlent pas d'une même voix» (Dorzee, 2000b : 17).

Enfin, les loueurs de kayaks reprochent au Ministre de ne pas avoir motivé sa décision sur base d'une "étude scientifique» ou d'un «élément objectif justifié». Par voie de presse, le Ministre a répondu avoir suivi l'avis de l'administration et s'être basé sur l'argument suivant: «Dans tous les cas évoqués, le niveau d'eau était insuffisant, la navigation devenait aléatoire. Il fallait trancher» (Dorzee, 2000b : 17).

Quant aux pêcheurs, à l'instar du Ministre, ils soulignent le caractère massif de l'activité en précisant que «les jours d'affluence, plus de 3000 kayaks descendent la basse Lesse entre Houyet et Anseremme ». Outre la massivité de l'activité décriée, c'est le caractère tumultueux et agité de la foule qui est également mis en exergue à travers l'utilisation de métaphores telles que «c'est la cohue comme en ville». Yves Rollin, président de l'ASBL Comité royal de l'amicale des pêcheurs de la Lomme et de la Lesse, ajoute : «Nous n'avons jamais été opposés au passage des kayakistes sportifs mais il y a une fameuse différence avec l'exploitation de masse que l'on constate actuellement» (Wilputte, 2000a : 15). Outre la massivité de l'activité décriée, c'est le caractère touristique qui est dénigré comparativement à l'activité sportive qui semble plus noble. Comme l'indiquent les quelques lignes suivantes, les techniques d'exploitation de la rivière font également partie des griefs reprochés aux kayak : «[A]vant l'entrée en vigueur de la loi qui l'interdit, les exploitants mettaient aussi des engins dans le lit des rivières pour débiter les arbres, déracinés certes, mais aussi pour détourner le cours des méandres, obtenir un débit plus rapide et faciliter le passage des barques. Au grand dam des pêcheurs et des naturalistes qui voient des radiers à frayères s'étioler, les rives s'éroder, les stries sauvages disparaître et les berges de la rivière se gorger de déchets...» (Haquin, 2000 : 21).

Ces quelques passages traduisent la manière dont les kayakistes, à qui l'on attribue des pratiques non respectueuses de l'environnement, sont perçus par les pêcheurs et les naturalistes en Wallonie (IEW, 2000b).

Enfin, les associations environnementales et de protection de la nature, par la voix de leur Fédération wallonne (InterEnvironnement Wallonie), soutiennent la décision du Ministre 
par voix de presse en mettant l'accent sur l'impact positif de ces mesures par rapport à l'équilibre entre les différents usages des cours d'eau : «Les cours d'eau wallons font partie du patrimoine régional et à ce titre, n'en déplaise à certains, leur gestion doit se faire dans l'intérêt collectif. La décision de limiter les périodes d'accès de [six] rivières wallonnes aux kayaks est une décision courageuse, car elle rétablit l'équilibre où les pêcheurs et les naturalistes étaient perdants ».

Nonobstant le principe que les différents usages de la rivière doivent se côtoyer "harmonieusement», InterEnvironnement Wallonie estime que la grande affluence de kayaks certains jours, perçue comme une «surexploitation touristique», "nuit à la biologie du cours d'eau et au tourisme lui-même». La Fédération considère que la protection du patrimoine et le tourisme ne sont pas incompatibles, mais appelle à une maîtrise des loisirs aquatiques, "opportunité que les loueurs de kayaks doivent saisir», en s'inscrivant dans la voie de la diversification.

Par ailleurs, Inter-Environnement Wallonie souligne que les rivières wallonnes sont fréquentées par les kayakistes des pays voisins, ce qui est amené comme un argument supplémentaire pour établir une réglementation stricte afin d'éviter que la Wallonie ne devienne «un parc de loisirs où tout est permis» et pour protéger son patrimoine naturel. La Fédération insiste dès lors pour que d'autres mesures de régulation voient le jour, qui, théoriquement, «devraient être notamment favorables aux loueurs de kayaks» (immatriculation des kayaks, autorisations environnementales pour la location, concertation entre les acteurs du secteur, contrôle renforcé de la circulation) et permettraient de "garantir une utilisation rationnelle, écologique et économique des rivières wallonnes» (IEW, 2000c).

Cette dernière affirmation d'Inter-Environnement Wallonie exprime bien les enjeux auxquels sont et seront de plus en plus confrontées les activités sportives et touristiques en milieu rural et naturel. Ces enjeux, liés à la multiplicité des usages de la rivière, et plus globalement de ceux de l'espace rural, ne sont cependant pas spécifiques à la Wallonie. En effet, ces trois dernières décennies, les fonctions de l'espace rural, tant de la Wallonie que de nombreux autres territoires occidentaux, ont connu de grandes mutations socio-économiques et culturelles. Ces espaces servent actuellement de support à quatre types de fonctions principales : économique (production agricole, tourisme), résidentielle (habitat permanent ou secondaire), écologique (protection du patrimoine naturel) et culturelle (conservation du patrimoine rural). Ces multiples fonctionnalités, qui induisent des modalités d'usage parfois concurrentes, sont régulièrement sources de conflits entre différents usagers qui désirent occuper l'espace rural et qui y projettent des usages et des représentations différentes. Des tensions peuvent donc naître de ces visions et comportements parfois incompatibles (Caron et Torre, 2002 : 96). Comme le suggère Mormont, ces incompatibilités entre usages opposent en réalité des groupes sociaux dans leurs démarches respectives d'appropriation de l'espace rural (Mormont, 2006 : 302).

En Wallonie, c'est précisément ce type de conflits qui se joue entre deux usages et représentations différentes de la rivière que sont, d'une part, une fonction plus économique symbolisée par les loueurs de kayaks et d'autre part, une approche plus écologique des cours d'eau revendiquée par les pêcheurs.

\section{Pêche et kayak en Wallonie : une illustration de l'usage de la rhétorique environnementale comme moyen de régulation de la fonction économique des cours d'eau}

Comme susmentionné, le kayak va connaître, dans les années 1985 à 1990, un fort engouement sur la basse Lesse, qui est le premier site d'implantation de cette activité en Wallonie. Cette époque correspond à celle d'une politique de promotion très intensive du kayak menée par des sociétés de location. L'augmentation de la pratique de cette activité sera également accentuée par l'essor du tourisme d'un jour et des loisirs et sports de nature. Cet engouement ne sera pas sans conséquences sur la qualité de navigation de la Lesse. Les conflits entre certains loueurs de kayaks et pêcheurs vont notamment inciter une grande entreprise de location à s'étendre sur un autre tronçon de la Lesse. Or, le tronçon en question (situé sur la haute Lesse) n'est pas adapté à la circulation massive de kayaks, car son débit est bien moindre qu'en aval. De par son activité, la présence de cette société de location va créer une forte contestation de la part des pêcheurs qui, regroupés en association, iront jusqu'au Conseil d'État (la plus haute instance administrative du pays), et obtiendront gain de cause en faisant annuler la décision qui autorisait la circulation sur ce tronçon en été. La fréquentation massive de la Lesse, souvent qualifiée "d'autoroute de kayaks», va contribuer fortement à ternir l'image de l'activité de location. Cette situation critique particulière, qui fit grand bruit au sein de la presse locale, régionale et nationale et qui peut être considérée comme l'un des facteurs qui a incité l'administration wallonne à renforcer la réglementation de l'activité, illustre ce que Mounet appelle une «montée en généralité abusive», définie comme une affirmation générale résultant de la transformation de "résultats locaux, périodiques et partiels» (Mounet, 2007b : 164).

Faisant face à des conflits d'usage de plus en plus importants opposant pêcheurs, praticiens du kayak et défenseurs de la nature, la Région Wallonne va donc renforcer la réglementation relative à la circulation des kayaks, et ce à plusieurs reprises, durant les années 1990 . Les autorités ont ainsi opté pour un modèle «autoritaire» de régulation, à savoir que les mesures de restriction ont été inscrites dans un cadre juridique dont le non respect, par définition, est passible de sanctions.

Plusieurs facteurs expliquent l'adoption du modèle réglementaire face aux conflits d'usage relatifs aux cours d'eau en Région wallonne, par rapport à d'autres modèles de régulation axés davantage sur la participation volontaire des acteurs en conflit. D’une part, depuis les années 80, mais surtout depuis la Conférence de Rio de 1992, on observe en Europe une multiplication des directives environnementales, et donc une augmentation des contraintes liées à l'usage des ressources naturelles. Ce contexte est ainsi prégnant en Région Wallonne, où les mesures législatives relatives à la gestion de différents domaines environnementaux se multiplient (eau, déchets, forêts, etc.). 
D'autre part, depuis les années 1970, les pêcheurs — bien implantés dans la vie politique locale — et les naturalistes exercent un lobbying important sur les autorités en matière de protection de la nature. La pêche est en effet un loisir de proximité et est donc fortement pratiquée par des locaux alors que le kayak est plus l'apanage de touristes et excursionnistes extra-locaux qui entretiennent de ce fait des relations davantage temporaires et occasionnelles avec les destinations touristiques et ses acteurs. Dès lors, de par leur réseau de relations locales, les pêcheurs sont davantage capables de peser sur les décisions politiques et réglementaires.

Renforcées par les discours axés sur l'environnement, les attentes des pêcheurs et naturalistes seront ainsi largement entendues par les pouvoirs publics durant les années 90, et notamment en ce qui concerne la régulation des conflits d'usage avec les kayaks. Leur point de vue sera d'autant plus écouté que le ministre Happart est un homme politique dont la proximité avec le milieu des pêcheurs est notoire.

Ainsi que nous l'avons mis en évidence au travers de l'analyse de la presse, le renforcement de la réglementation en 2000 sera la mesure de trop aux yeux des loueurs de kayaks. Les décisions prises ont en effet été mal acceptées par les loueurs de kayaks et ont suscité une polémique. Cette polémique oppose, d'une part, les loueurs de kayaks, qui craignent des pertes économiques importantes pour le secteur et, de l'autre, le ministre Happart, qui a pris la décision au titre de la protection de l'environnement et de l'équilibre des usages de la rivière, ainsi que les pêcheurs et les associations environnementales qui partagent son point de vue. Nous observons ainsi la répétition d'une résolution de conflit défavorable aux loueurs de kayaks, résultant d'un rapport de force inégal en faveur des pêcheurs et des associations environnementales.

Au-delà des multiples impacts que les mesures vont causer au secteur (manques à gagner, fermetures d'entreprises de location, délocalisation vers d'autres cours d'eau, image), le manque de légitimité de ces mesures, marqué par l'incompréhension des loueurs de kayaks, est à attribuer, selon nous, à deux facteurs majeurs. D'un côté, les décisions ont été prises en l'absence d'arguments scientifiques valables. L'impact négatif du kayak sur les biotopes est en effet une justification avancée par les pouvoirs publics pour motiver les mesures au titre de la protection de la nature. Cependant, cet impact n'a pas été prouvé par une étude scientifique reconnue comme telle par les différents acteurs. D'un autre côté, la concertation entreprise par le Ministre avant d'adopter les mesures n'a pas du tout été considérée satisfaisante aux yeux des loueurs de kayaks, qui estiment ne pas avoir pu faire valoir leur point de vue.

\section{L'incertitude scientifique, source de controverse}

En ce qui concerne l'absence d'argumentation scientifique, plusieurs éléments nous permettent d'avancer l'existence d'une controverse scientifique dans le contexte de l'activité du kayak en Wallonie, ainsi que Mounet l'a observé dans différentes situations de conflits d'usage liés aux sports de nature (Mounet, 2007a).

En effet, le Ministre a affirmé dans la presse s'être basé sur l'avis de l'administration, mais, au sein de celle-ci, les fonctionnaires ne font état d'aucune étude disponible et utilisée à ce jour. Administration et Ministre se renvoient donc la responsabilité de l'argumentation. D'un point de vue scientifique, on peut également observer l'absence d'étude de terrain sur les impacts environnementaux des loisirs et sports de nature. Les seules études actuellement disponibles en Wallonie portent sur une caractérisation générale qualitative et non spatialisées des impacts environnementaux des loisirs sportifs de nature (De Myttenaere et al. 2007 : 116).

Au regard de la controverse que suscite un tel contexte d'incertitude scientifique, nous identifions plusieurs facteurs qui peuvent avoir été déterminants dans la décision du Ministre. La volonté de réduire des impacts potentiels, point commun à de nombreuses politiques environnementales, pourrait être avancée comme étant à l'origine de la décision ministérielle. Cependant, la prégnance des rapports de force entre loueurs de kayaks et pêcheurs rend possible l'argument selon lequel l'incertitude scientifique a été «manipulée par les acteurs» et que l'impact environnemental de l'activité a été "confondu, volontairement ou non, avec le conflit d'usage», situation considérée comme étant courante dans les conflits d'usage (Mounet, 2007b : 164).

À l'origine de cet amalgame, les présupposés idéologiques jouent un rôle important. Selon nous, le kayak hérite de l'image négative et de la stigmatisation fréquente des rapports qu'entretiennent le tourisme et les loisirs de nature avec l'environnement. En effet, il n'est pas rare d'observer dans des dossiers de presse, des communiqués d'associations environnementalistes ou dans la littérature scientifique une dénonciation des impacts, toujours présupposés négatifs, que provoqueraient les activités touristiques dans les territoires concernés par ces activités. Pêle-mêle, le tourisme y est accusé de provoquer des destructions d'écosystèmes, de favoriser le pillage de réserves d'eau, de banaliser et pervertir les lieux, de dénaturer ou détruire les paysages, d'exacerber les compétitions pour l'accès aux ressources entre les usagers locaux et les touristes (conflits d'usage pour l'utilisation des terres, de l'eau, de l'énergie, etc.). À en croire cette littérature, le tourisme serait systématiquement responsable de nombreux maux.

Quant à la pêche, elle est par contre communément perçue comme respectueuse de l'environnement et bénéficie d'une image plus positive. Pourtant, l'activité de pêche n'est pas sans incidences pour l'environnement de par certaines techniques reconnues perturbatrices pour les milieux (rempoissonnement, piétinement et tassement des berges, abandon de détritus, etc.) (Jouret, $2000: 37$ ).

Par ailleurs, il n'existe pas en Wallonie d'étude scientifique permettant de confirmer ou d'infirmer que la pêche serait plus respectueuse de l'environnement que ne le serait la pratique du kayak. Dès lors, l'abondance et la grande variété d'idées reçues négatives sur le tourisme, ainsi que l'hostilité que certains milieux lui témoignent expliquent partiellement, selon nous, les restrictions adoptées par le Gouvernement au nom de la conservation de la nature. La rhétorique environnementale apparaît donc, dans ce cas de figure, comme un moyen qui vient délégitimer un mode d'usage de l'espace par rapport à un autre. 


\section{Les loueurs du kayak inorganisés}

\section{face au puissant lobbying des pêcheurs}

L'incapacité des loueurs de kayaks à faire valoir leur propre intérêt dans la prise de décisions politiques, comparativement à d'autres acteurs comme les pêcheurs, permet d'expliquer l'existence de rapports de force inégaux en faveur d'une certaine représentation de l'intérêt environnemental et en défaveur de l'intérêt économique.

Nous avons vu que les loueurs de kayaks ont vivement réagi dans la presse et dénoncé un manque de concertation avec les autorités régionales. Les concertations organisées par le gouvernement wallon se seraient en effet «apparentées à un dialogue de sourds, la décision du ministre étant déjà prise et inflexible» (FLKW, 2000). Le Ministre a, en réaction, déploré l'absence d'un interlocuteur représentatif des revendications des loueurs de kayaks. Il est vrai que la Fédération des loueurs de kayaks en Wallonie (FLKW) aurait pu endosser cette responsabilité, mais il semble que de fortes discordes internes n'aient pas facilité le dialogue pourtant si nécessaire aux regards des enjeux qui concernent le secteur.

Pourtant, la FLKW a cherché à réorganiser le secteur, consciente d'une part, du caractère atomisé de l'ensemble des acteurs des activités sportives de nature, et d'autre part, d'une certaine mauvaise image de son activité du point de vue de la protection de l'environnement. Cela a pris la forme d'un projet de fédération regroupant toutes les entreprises de tourisme nature et d'aventure de la Région wallonne. Cherchant à prendre le rôle d'interlocuteur représentatif, cette fédération vise à mieux réglementer, et donc crédibiliser, la cinquantaine de disciplines qu'elle rassemble, à les responsabiliser et à faire reconnaître le secteur à part entière, en vue de pouvoir fonctionner en véritables réseaux commerciaux ou d'influence pour notamment bénéficier du soutien financier de l'administration du tourisme, au même titre que les attractions touristiques ou l'hôtellerie.

Cependant, ce projet de fédération n'a finalement pas vu le jour, notamment du fait de l'existence de fortes divergences de vue et de tensions entre les petites et les grandes entreprises du secteur du kayak. En effet, la réforme de 2000 a touché davantage les petites entreprises de location et épargné les grandes entreprises, dont la plupart se situent sur des tronçons de rivière dits «sacrifiés» et exploités commercialement depuis longtemps (Lesse et Ourthe). L'absence d'une réglementation encore plus stricte du secteur et le manque de contrôles les arrangeraient dès lors assez bien. Ces grandes entreprises n'auraient a priori aucun avantage à ce qu'apparaissent des quotas de kayaks par rivière, des chartes de bonne conduite, des contrôles plus appuyés...

Ces différents éléments nous incitent à affirmer que les loueurs de kayaks n'ont pas réussi à faire valoir leurs intérêts avant l'adoption des nouvelles mesures réglementaires et à peser sur la décision en raison d'un véritable manque de consensus au sein de leur secteur. Cette absence d'unité de point de vue a empêché ce groupe d'acteurs de pouvoir rééquilibrer les rapports de force entre leur groupe et celui des pêcheurs.

Il résulte de cette analyse que le conflit observé entre les pêcheurs et les kayakistes en Wallonie est également un conflit symbolique et politique. En effet, ce qui a joué un rôle décisif pour l'issue du conflit opposant les kayakistes aux pêcheurs, c'est bien la capacité de ces derniers d'imposer comme catégorie légitime, leur représentation de l'espace rural comme un espace à protéger en priorité.

\section{Conclusion}

Tendance importante de l'usage touristique de nombreux territoires, l'engouement pour les activités sportives de nature de ces trois dernières décennies s'inscrit dans un nouveau rapport au temps, au corps et à la nature au sein de la société occidentale. L'essor qu'a connu le kayak comme activité touristique en Wallonie depuis les années 1980-1990 ressort de ces mêmes logiques. Essentielle dans certaines communes et marginale dans d'autres, cette activité contribue aujourd'hui de manière non négligeable à l'image et à l'économie de certains territoires.

Comme dans de nombreuses régions, la pratique du kayak en Wallonie est entrée en conflit avec d'autres usages de la rivière, tels que la pêche. En effet, les pêcheurs considèrent que le kayak est une activité incompatible avec la leur et reprochent souvent aux touristes pratiquant le kayak d'être bruyants et peu soucieux de l'environnement. À contrario, les pêcheurs se présentent comme détenteurs d'une certaine expertise écologique et fervents défenseurs de la nature, ce qui leur octroie une image positive et un pouvoir de négociation non négligeable.

En Wallonie, la rivière est ainsi le théâtre de conflits d'usage qui opposent des groupes d'acteurs aux valeurs et intérêts souvent divergents. Dans ce contexte, force est de constater que la pratique du kayak a été touchée par de nombreuses réglementations et soumise à davantage de contraintes. Perçues comme injustifiées par les loueurs de kayaks, ces mesures ont rapidement suscité des polémiques alimentant avec abondance la presse locale et régionale. En effet, l'absence d'étude objectivant les impacts environnementaux de la pratique du kayak a généré de fortes controverses. Devant cette situation, cet article visait à identifier les éléments qui expliquent l'application de mesures réglementaires par les autorités régionales, perçues comme particulièrement injustes et illégitimes par les loueurs de kayaks.

Plusieurs facteurs ont été mis en évidence : une forte tendance à la diabolisation des rapports du tourisme avec l'environnement, une généralisation abusive de situations particulières, ainsi que l'exercice d'un lobbying important par certains groupes sociaux tels que les pêcheurs et naturalistes.

L'influence des pêcheurs apparaît d'autant plus importante qu'elle est renforcée par une incapacité des loueurs de kayaks à faire valoir leurs propres intérêts lors de l'élaboration des mesures de régulation. Cette difficulté d'organisation et de négociation des loueurs de kayaks, attribuable notamment à des divergences de vues et à des tensions entre les petites et les grandes entreprises de location, est une des causes de l'échec récent d'un projet de création d'une fédération regroupant l'ensemble des opérateurs touristiques d'activités de loisirs de nature et d'aventure.

Dans un contexte où les activités ludosportives occupent une place de plus en plus importante au sein de nombreux 
territoires touristiques, l'absence d'une telle fédération reconnue par les autorités publiques constitue un facteur très défavorable vis-à-vis de la capacité du secteur à pouvoir fonctionner en véritables réseaux commerciaux ou d'influence, et à défendre d'une seule voix leurs intérêts face à d'autres acteurs mieux relayés et positionnés politiquement, et bénéficiant d'une meilleure image.

L'exemple du secteur de la location de kayaks démontre qu'en l'absence d'une objectivation scientifique des enjeux environnementaux, les rapports de force entre acteurs orientent fortement le contenu des réglementations, particulièrement dans une région comme la Wallonie où le modèle réglementaire constitue une option privilégiée pour arbitrer entre la protection de l'environnement et les différents usages du milieu rural. De fait, les conflits locaux observés en Wallonie entre les pêcheurs et les loueurs de kayaks ne sont pas seulement la manifestation de conflits d'intérêts (culturels et symboliques), mais également celle d'un processus dans lequel s'élaborent et se mettent en forme des argumentations environnementales et des coalitions sociales qui vont conduire à des normes légales arbitrant in fine le rapport de force entre les parties en présence. Le cas du kayak en Wallonie indique donc que lorsqu'une représentation de l'espace arrive à s'imposer, il en résulte que certaines catégories sociales peuvent en être totalement ou partiellement exclues.

Note

1 Les auteurs tiennent à remercier, pour leur précieuse collaboration à cet article, Marc Nielsen (cartographie) et Jérémie Dandois (travaux d'enquête).

\section{Bibliographie}

BERTRAND, Michel (2000) «Les loueurs de kayaks se jettent à l'eau », $L a$ Dernière Heure, 4 août, p. 7.

BESSY, Olivier et Michel MOUTON (2004) «Du plein air au sport de nature. Nouvelles pratiques, nouveaux enjeux», Cahier Espaces, $n^{\circ}$ 81, "Sports de nature. Évolutions de l'offre et de la demande», mai 2004, p. 13-29.

CARON, Armelle et André TORRE (2002) «Les Conflits d'usages dans les espaces ruraux. Une analyse économique», Sciences de la Société, n 57 , p. 95-113.

Cellule état de l'environnement wallon (2008) Tableau de bord de l'environnement wallon, Service Public de Wallonie, Direction générale opérationnelle Agriculture, Ressources naturelles et environnement, Région Wallonne.

CORNELOUP, Jean; Malek BOUHAOUALA; Cécile VACHÉE et Bastien SOULÉ (2001) «Formes de développement et positionnement touristique des espaces sportifs de nature», Loisir et Société, vol. 24, $\mathrm{n}^{\circ} 1$, p. 21-46.

DE GENDT, Pascal (2000) «Les loueurs de Kayaks ne baissent pas les bras», $L a$ Libre Belgique, 4 août, p. 4.

DE MYTTENAERE, Bernard; Emmanuel D'IETEREN et Marie-Françoise GODART (2007) Le tourisme et les loisirs, dans CEEW, Rapport analytique sur l'état de l'environnement wallon 2006-2007, Namur, Cellule état de l'environnement wallon, MRW-DGRNE, p. 105-119.

DEPREST, Florence (1997) Enquête sur le tourisme de masse : L'écologie face au territoire, Paris, Belin, $207 \mathrm{p}$.

DGARNE - Direction générale agriculture, ressources naturelles et environnement (2009), Communications personnelles.
DORZEE, Hugues (2000a) «Le kayak écarté des eaux wallonnes», Le Soir, 28 octobre, p. 19.

DORZEE, Hugues (2000b) «Coups de rame wallonne contre l'arrêté «cours d'eau»", Le Soir, 5 août, p. 17.

ERNENS, Catherine (2001) «Le dernier été des kayaks», Vers l'Avenir, 20 juillet, p. 5.

LEKANE, Eric (2000) «Les réformes de José Happart font des vagues», Vers l'Avenir, 31 juillet, p. 3.

FLKW - Fédération des loueurs de kayaks en Wallonie (2000), Communiqué de presse, 4 août.

FONTUGNE, Christian (2004) «Les sports de nature. Un outil de structuration de l'offre touristique», Cahier Espaces, $n^{\circ} 82$, «Sports de nature. Des territoires et des hommes», juillet 2004, p. 15-19.

GÉRARD, Pierre et Benoit DE BAST (2000) « Restriction of the circulation of small pleasure boats on the rivers of Wallonia ", Fisheries Management and Ecology, Belgium, 2000, n ${ }^{\circ}$, p. 139-143.

Gouvernement wallon (2000) Communiqué de presse, 26 octobre.

HALLET, Catherine et An WOUTERS (1992) «Le tourisme en Wallonie : quels défis?», Revue Environnement, $\mathrm{n}^{\circ}$ 13, Dossier tourisme «Wallonie : terre d'accueil, terre d'écueil», juin 1992, p. 4-6.

HAQUIN, René (2000) «Kayaks: Happart maintient le cap », Le Soir, 9 juin, p. 21.

HENRARD, Jean-Luc (2000) «Les kayaks ne veulent pas couler», Viroinval, 16 juin.

IEW - Inter-Environnement Wallonie (2000a) «La nature a aussi son mot à dire», Communiqué de presse, 10 août.

IEW - Inter-Environnement Wallonie (2000b) Le développement des sports «nature» en Région wallonne, Inter-Environnement Wallonie, février 2000.

IEW - Inter-Environnement Wallonie (2000c) «Happart va dans le bons sens», Communiqué de presse, 5 mai.

JOURET, Patrick (2000) Sport et environnement. Sport et Société, Réflexion prospective, Atelier 50, Rapport à la Fondation Roi Baudouin, Bruxelles, Centre de diffusion - Fondation Roi Baudouin, 48 p.

KNAFOU, Rémy et Équipe MIT (2002) Tourismes 1. Lieux communs, Équipe de recherche MIT (Mobilités, Itinéraires, Territoires) de l'Université Paris-VII, Paris, Belin, 320 p.

MORMONT, Marc (2006) "Conflits et territorialisation », Géographie, Économie, Société, n 8, p. 299-318.

MOUNET, Jean-Pierre (2007a) «La gestion environnementale des sports de nature : entre laisser-faire, autorité et concertation ", Développement durable et territoire, Varia, mis en ligne le 11 juin 2007. <http://developpementdurable.revues.org/document3817.html> (Consulté le 3 septembre 2008)

MOUNET, Jean-Pierre (2007b) «Sports de nature, développement durable et controverse environnementale», Natures Sciences Sociétés, $\mathrm{n}^{\circ}$ 15, p. 162-166.

MOUNET, Jean-Pierre (2000) Les activités sportives de nature en France: contraintes globales, flou organisationnel et stratégies d'acteurs, Diplôme d'habilitation à diriger des recherches de l'Université J. Fourier de Grenoble, Laboratoire EROS (JE 540), soutenu le 3 juillet.

MOUNET, Jean-Pierre (1997) «Les activités physiques et sportives dans l'offre touristique. De l'utilité d'une mise en réseau et d'une labellisation ", Cahier Espaces, $\mathrm{n}^{\circ}$ 52, «Tourisme et sport», septembre 1997, p. 102-110.

PAOLI, Jean-Christophe (2008) Typologie des conflits sur l'espace en fonction des institutions régulatrices : essai sur un échantillon relaté par la presse quotidienne régionale en Corse, dans Thierry Kirrat et André Torre (ed.), Territoires de conflits, Paris, L'Harmattan, $322 \mathrm{p}$. 
PERRIN, Clémence et Jean-Pierre MOUNET (2004) «L'insertion de l'offre de canoying dans l'industrie touristique : une étude comparative entre le Massif des Bauges et le Vercors», Loisir et Société, vol. 27, n 1, p. 143-172. REVÉRET, Christophe (2004) «Le discret Big Bang des loisirs sportifs de nature», Cahier Espaces, ${ }^{\circ}$ 82, «Sports de nature. Des territoires et des hommes», juillet, p. 8-14.

R. P. (2000) «Des kayaks indésirables», La Dernière Heure, 26 juillet, p. 10. RW - Région wallonne (non daté) «Kayak - Circulation sur les cours d'eau », Région wallonne, <http://kayak.environnement.wallonie.be/ index.htm>.

THEYS, Jacques (2003) «La Gouvernance, entre innovation et impuissance, Développement durable et territoire», Dossier 2 : Gouvernance locale et développement durable, mis en ligne le $1^{\text {er }}$ novembre 2003. <http:// developpementdurable.revues.org/document1523.html> (Consulté le 3 septembre 2008)

TORRE, Alain et Christine LEFRANC (2006) «Les conflits dans les zones rurales et périurbaines. Premières analyses de la presse Quotidienne Régionale», Espaces et Sociétés, vol. 124-125, p. 93-110.
VANDERMOTTEN, Christian; Alain COLLARD; Lucia GAIARDO et Dominique ISTAZ (1995) Tourisme, dans Ministère de la Région wallonne, État de l'Environnement Wallon 1994. Volume 1, Jambes, Ministère de la Région wallonne, Direction générale des Ressources naturelles et de l'environnement, $467 \mathrm{p}$.

VIGARELLO, Georges (1982) «Le corps...entre illusions et savoirs», revue Esprit, 1982.

WILPUTTE, Emmanuel (2003), «Pour redorer le blason des kayaks», Vers l'Avenir, 20 mars 2003, p. 21.

WILPUTTE, Emmanuel (2001) «Pêcheurs 1, loueurs de kayak 0 », Vers l'Avenir, 14 février, p. 21.

WILPUTTE, Emmanuel (2000a) «Des entreprises en péril en Haute-Lesse et sur le Viroin», Vers l'Avenir, 5 août, p. 15.

WILPUTTE, Emmanuel (2000b) «Bataille perdue dans la guerre des kayaks», Vers l'Avenir, 1 août, p. 15.

\section{Appel à textes}

En dehors des dossiers thématiques, Téoros ouvre ses pages aux propositions spontanées. La rédaction invite tous les chercheurs qui œuvrent dans le domaine du tourisme ou qui s'intéressent au tourisme sous tous ses aspects à soumettre des articles de nature analytique à la revue. On peut soumettre un article en l'envoyant à :

\section{teoros@uqam.ca}

Les textes soumis doivent apporter une contribution scientifique originale, que ce soit par le biais d'information factuelle jusqu'alors inconnue ou par une nouvelle interprétation d'un thème particulier. Téoros vise avant tout le transfert de connaissances; son objectif est donc de promouvoir une meilleure compréhension des phénomènes liés au tourisme.

Les auteurs doivent faire parvenir un manuscrit présenté selon les règles de la revue, disponibles au www.teoros.uqam.ca. Un article analytique compte environ 6000 mots et n'excède pas
8000 mots, avec trois ou quatre illustrations en 300 ppp. Les articles peuvent être soumis en anglais ou en français et doivent être accompagnés d'un résumé de 200 mots et de cinq mots-clés.

La publication des articles se fait sous réserve d'une évaluation. Tous les manuscrits seront évalués anonymement par des pairs qui pourront faire des suggestions ou demander des modifications. La rédaction transmettra l'avis des évaluateurs aux auteurs et s'assurera que les modifications demandées seront apportées.

Au plaisir de vous lire dans nos pages.

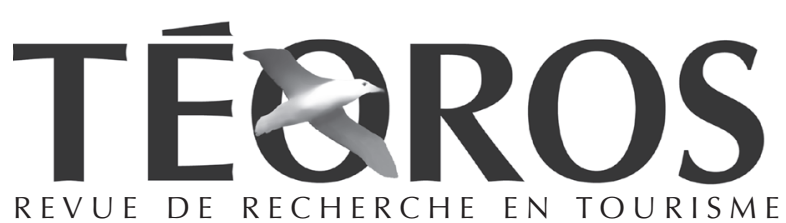

